\section{OP0056 PLASMA MICROVESICLES AS LIQUID BIOPSIES OF THE ARTERIAL WALL IN LARGE VESSEL VASCULITIS}

E. Tombetti ${ }^{1}$, K. H. Chun ${ }^{2}$, C. lannone ${ }^{3}$, E. M. Baldissera ${ }^{4}$, J. Mason ${ }^{5}$, A. Manfredi ${ }^{6}{ }^{1}$ Milan University, Biomedical and Clinical Sciences Luigi Sacco, Milan, Italy; ${ }^{2}$ Vita-Salute San Raffaele University, Milan, Italy; ${ }^{3}$ Vita-Salute San Raffaele University, Autoimmunity and Vascular Inflammation, Milan, Italy; ${ }^{4}$ Ospedale San Raffaele, UniRAR, Milan, Italy; ${ }^{5}$ Hammersmith Hospital, Vascular Sciences and Rheumatology - NHLI, London, United Kingdom; ${ }^{6}$ Vita-Salute San Raffaele University, Autoimmunity and Vascular Inflammation; UniRAR, Milan, Italy

Background: Large-vessel vasculitides comprise Takayasu arteritis (TA)and giant cell arteritis (GCA). Arterial stenosis and dilatation directly affect prognosis but the mechanism(s) underlying remodeling of the vessel wall have not been identified. Microvesicles (MVs) are membrane-enclosed extracellular vesicles released upon cellular activation and stress and as a consequence of environmental inflammation. MVs maintain features and constituents of their parental cells. They have been proposed to serve as potential liquid biopsies in oncology. Objectives: To verify whether arterial wall derived-MVs are recognizable in the blood of TA patients and express bioactive molecules potentially involved in arterial injury, inflammation and remodeling.

Methods: Platelet was obtained from 112 LVV pts (73 TA, 39 GCA), 42 age and age- and sex-matched healthy controls $(\mathrm{HC})$ and 30 pts with severe carotid atherosclerosis requiring vascular surgery. Plasma flow cytometry was performed with anti-CD14, CD16, anti-CD144 (VE-cadherin, an endothelial marker), anti-CD140a/b (PDGF receptor A/B a vascular stromal marker), anti-HMGB1, anti-PTX3, mitotracker green (that identifies mithochondrial moieties) and mitosox (that revels mitochondrial reactive oxygen species). MVs were identified by physical parameters using Gigamix beads. Medium- to large-sized MVs were defined as MVs with $>240 \mathrm{~nm}$-eq diameter. Results: Preliminary results are available for 49 LVV (42 TA, 7 GCA), 8 severe carotidatherosclerosis and 14 age- and sex-matched $\mathrm{HC}$. As compared to $\mathrm{HC}$ or $\mathrm{CA}, \mathrm{LVV}$ plasma contains a higher number of MVs and in particular of medium- to large- sized MVs ( $p<0.001$ for all comparisons) (Figure, panels A-B). Next, we evaluated the MVs surface expression of markers of leukocytic, endothelial and stro$\mathrm{mal} / \mathrm{vascular}$ stromal lineages. Total counts of CD14+, CD16+, CD66b+, CD140a+, $\mathrm{CD} 140 \mathrm{~b}+, \mathrm{CD} 144+\mathrm{MVs}$ were increased in LVV plasma with very high level of significance (Figure, panels C-G) while higher percentage of CD16+ and CD140a+ medium-to large-sized MVs was found in atherosclerosis. Expression of molecules involved in inflammation or repair, PTX3 or HMGB1 mitochondrial antigens and mitochondrial ROS all were consistently higher in LVV (Figure, panels H-M).

Conclusion: MVs, including those expressing arterial stromal biomarkers, are increased in LVV plasma, suggesting a communication between the vessel wall and peripheral blood. MV express signals that may in turn contribute to persisting vascular inflammation in large vessel vascultis Further analysis is required to dissect their potential use as disease biomarkers
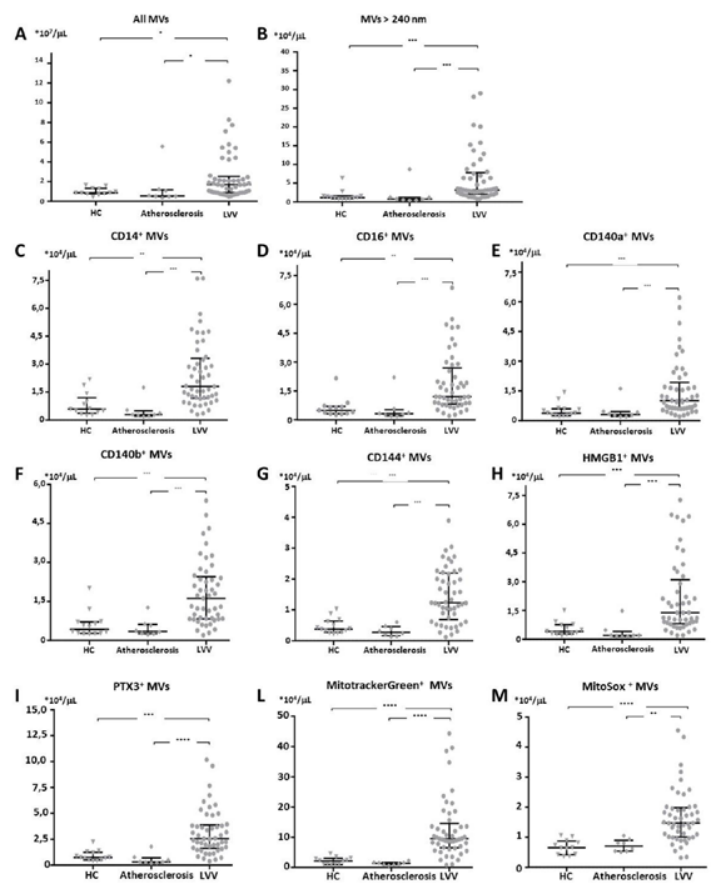

REFERENCES:

[1] van Niel G et al, Nat Rev Mol Cell Biol. 2018

[2] Mason JC. Nat Rev Rheumatol. 2010

Disclosure of Interests: None declared

DOI: 10.1136/annrheumdis-2021-eular.4300

\section{OP0057 A PERSONALISED RITUXIMAB RETREATMENT APPROACH BASED ON CLINICAL AND B-CELL BIOMARKERS IN ANCA-ASSOCIATED VASCULITIS}

J. Arnold ${ }^{1}$, E. Vital ${ }^{1}$, S. Dass ${ }^{1}$, A. Aslam ${ }^{1}$, A. Rawstron ${ }^{2}$, S. Savic ${ }^{1}$, P. Emery ${ }^{1}$, M. Y. MD Yusof ${ }^{1} .{ }^{1}$ Chapel Allerton Hospital, Leeds Institute of Rheumatic and Musculoskeletal Medicine, Leeds, United Kingdom; ${ }^{2}$ St James's University Hospital, Haematological Malignancy Diagnostic Service, Leeds, United Kingdom

Background: Time-to-relapse after rituximab for ANCA-associated vasculitis (AAV) is variable and optimal retreatment strategy has been unclear. We previously showed that repopulation of naïve B-cells at 6 months predicts sustained response [1].

Objectives: In AAV following rituximab induction, to evaluate clinical and B-cell predictors of relapse in order to develop a retreatment algorithm.

Methods: An observational study was conducted in 60 rituximab-treated AAV patients followed for over 10 years. Complete response (CR) was defined as Birmingham Vasculitis Activity Score v3.0 $=0$. Retreatment was given on clinical relapse, defined as new features or worsening of persistent disease (not by biomarker status). Peripheral B-cell subsets were measured using highly sensitive flow cytometry. Predictors were tested using multivariable Cox-Regression.

Results: Median times-to-retreatment for rituximab cycles $1-5$ were $87,71,65$, 59 and 86 weeks. Over 417 patient-years follow-up, 137 relapses occurred in 50 patients; 16 (in 14 patients) were major (renal=7, neurological=4, ENT=3 and respiratory $=2$ ). The major-relapse rate was $3.8 / 100$ patient-years. In multivariable analysis, concomitant immunosuppressant [HR 0.48 (95\% Cl 0.24-0.94)], achieving CR $[0.24(0.12-0.50)]$ and naïve B-cell repopulation at 6 months $[0.43$ $(0.22-0.84)]$ were associated with longer time-to-relapse. Higher baseline memory B-cells [1.01 (1.00-1.02)] were associated with a shorter time-to-relapse. AUROC for prediction of time-to-relapse was greater if guided by naïve B-cell repopulation than if ANCA and/or CD19+ return at 6 months had been used, 0.82 and 0.52 respectively.

Conclusion: These data suggest that all patients should receive concomitant oral immunosuppressant. Those with incomplete response or with absent naïve B-cells should be retreated at 6 months. Patients with complete response and naïve repopulation at 6 months should not receive fixed retreatment. This algorithm could reduce hypogammaglobulinaemia due to unnecessary retreatment.

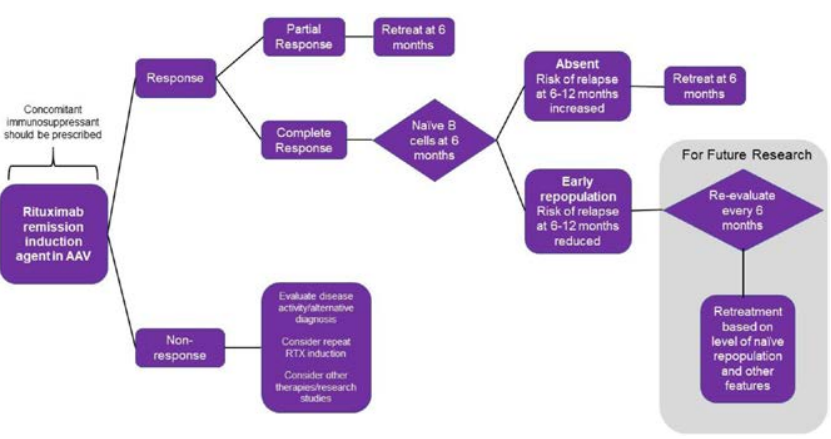

Figure 1. A personalised retreatment algorithm for rituximab in ANCA-associated vasculitis

\section{REFERENCES:}

[1] Md Yusof et al. Annals of rheumatic diseases (2015) PMID: 25854586. Disclosure of Interests: Jack Arnold: None declared, Edward Vital Speakers bureau: Roche, GSK and AstraZeneca, Consultant of: Roche, GSK and AstraZeneca, Grant/research support from: Roche, GSK and AstraZeneca, Shouvik Dass Speakers bureau: Roche and GSK, Aamir Aslam: None declared, Andrew Rawstron: None declared, Sinisa Savic: None declared, Paul Emery Speakers bureau: BMS, Abbott, Pfizer, MSD, Novartis, Roche and UCB, Consultant of: BMS, Abbott, Pfizer, MSD, Novartis, Roche and UCB, Grant/research support from: Abbott, BMS, Pfizer, MSD and Roche., Md Yuzaiful Md Yusof: None declared

DOI: 10.1136/annrheumdis-2021-eular.2193 